\title{
ASPECTOS ECOLÓGICOS DE COMUNIDADES BÉNTICAS DE UN SISTEMA FLUVIAL ANDINO, ARMENIA - QUINDÍO, COLOMBIA
}

\section{ASPECTS ECOLOGICAL OF BENTHIC COMMUNITIES IN A FLUVIAL ANDEAN SYSTEM, ARMENIA - QUINDÍO, COLOMBIA}

\author{
Nidia Carolina Marín Villegas ${ }^{1}$, Nancy Viviana Corredor Coy ${ }^{1}$, César Augusto Bustamante Toro ${ }^{2}$ \\ ${ }^{1}$ Egresadas Programa académico Licenciatura en Biología y Educación Ambiental. Universidad del Quindío. E-mail: caromarin13@gmail.com , vivianacorredor@gmail.com \\ 2 Programa académico Licenciatura en Biología y Educación Ambiental, Grupo de investigación BIOEDUQ. Universidad del Quindío. E-mail: ceabusta@uniquindio.edu.co \\ Fecha de recibido: Febrero 12 de 2011 \\ Fecha de aceptado: Junio 22 de 2011 \\ Correspondencia: Programa Licenciatura en Biología y Educación Ambiental. Universidad del Quindío Armenia Quindío Colombia.
}

\begin{abstract}
RESUMEN
Se estudiaron algunas comunidades bénticas en la quebrada La Florida Armenia-Quindío, durante un periodo de cinco meses comprendidos entre febrero y junio de 2009. Para ello, se establecieron seis puntos donde se colectaron muestras de macroinvertebrados y algas perifíticas en sustrato epilítico (roca) y epifítico (vegetal), y se tomaron muestras de agua para su análisis físico-químico. Para evaluar la estructura ecológica de las comunidades bénticas se realizó una curva de acumulación de especies y se aplicaron los índices de diversidad ecológica alfa y beta; se compararon los índices alfa entre sustratos, sitios y meses de muestreo empleando una prueba de Kruskall Wallis y una de medias. Con base a los resultados, la comunidad de macroinvertebrados registró: 4 clases, 11 órdenes, 25 familias y 32 géneros, con un total de 12.113 individuos; por su parte, la comunidad de perifiton registró: 4 divisiones, 26 familias y 42 géneros, con un total de 23.626 (ind/ml). Los macroinvertebrados presentaron cambios en su estructura en los meses de abril, mayo y junio considerados tradicionalmente periodos de transición hidroclimático, y el sustrato rocoso se constituyó en su hábitat preferido; mientras las algas perifíticas no registraron cambios en su estructura en los diferentes meses de muestreo y su hábitat preferido fue el material vegetal. Los índices ecológicos alfa $(\alpha)$ muestran que los sitios 1 y 2 presentaron buen estado ecológico lo cual permite albergar un buen número de especies bénticas, mientras los sitios 4, 5 y 6 presentaron sus habitas y paisaje deteriorados. Es fundamental que se tomen acciones estratégicas para el manejo integral de la quebrada La Florida por parte de las autoridades ambientales, para evitar un mayor deterioro de sus aguas y ambiente biofísico.
\end{abstract}

Palabras claves: Macroinvertebrados, Perifiton, Sustratos, Índices ecológicos, Quebrada la Florida.

\begin{abstract}
Some benthic communities were studied in the stream "La Florida" Armenia - Quindío, during a period of five months between February and June, 2009. For that purpose, six points of sampling were established to collect samples of macroinvertebrates and periphytics seaweeds in ephilitc substratum (rock) and epiphytic (vegetable); and samples of water were taken for physic-chemical analysis. To evaluate the ecological structure of the benthic communities, a species accumulation curve was made, and the ecological diversity alpha and beta indices were applied; the alpha index was compared among substratums, places and period of time sampling, using a Kruskall Wallis's test and another test based on averages. The results obtained showed that the macroinvertebrates community registered: 4 classes, 11 orders, 25 families and 32 genera with a total of 12.113 individuals. On the other hand, the periphyton community registered: 4 divisions, 26 families and 42 genera with a total of 23.626 (ind $/ \mathrm{ml}$ ). The macroinvertebrates presented changes in their structure in April, May and June, which are considered traditionally periods of transition, and the rocky substratum was constituted in their favorite habitat; while the periphytics seaweeds did not register changes in their structure in the different months of sampling, in addition, their favorite habitat was the vegetable material. The ecological alpha $(\alpha)$ index showed that the sites 1 and 2 presented a good ecological condition that is used as a shelter for a good number of benthic species, while the sites 4, 5 and 6 presented a habitat and landscape in very bad conditions. It is fundamental to take strategic actions to
\end{abstract}


restore the integral treatment of the stream "La Florida" by the environment pollution control authorities to avoid a major deterioration of its waters and biophysical condition.

Keywords: Macroinvertebrates, Periphyton, Substrates, Ecological index, La Florida stream.

\section{INTRODUCCIÓN}

Entre las comunidades bénticas que habitan los sistemas lóticos andinos están los macroinvertebrados que comprenden una gran parte de la diversidad biológica, siendo con frecuencia el principal componente animal de estos sistemas (Esteves, 1988, citado por Castellanos y Serrato, 2008); estos organismos juegan un papel importante en la red trófica controlando la cantidad y distribución de sus presas y constituyendo una fuente alimenticia para consumidores terrestres y acuáticos (Wade et al., 1989, citado por Castellanos y Serrato, 2008), además de acelerar la descomposición de detritos y contribuir al reciclaje de nutrientes (Wallace et al., 1997, citado por Castellanos y Serrato, 2008). Por otra parte, también se encuentran las algas del perifiton que constituyen una comunidad adherida a diferentes sustratos en un río y son los productores primarios de la red trófica (Allan, 1995; Wetzel 2001, citado por Castellanos y Serratos, 2008); esta comunidad es la encargada de la producción de metabolitos orgánicos para diversos organismos, posee una alta tasa de reciclaje de nutrientes, dado que en muchos organismos encuentran abrigo $u$ otros alimentos, y a su vez es considerada como indicadora de calidad del agua (Roldan 1992).

En las quebradas andinas colombianas se han realizado pocos estudios ecológicos sobre comunidades bénticas, entre ellos, el empleo de los macroinvertebrados para evaluar calidad del agua como los reportados por Roldán et al. (1973); Machado y Roldán (1981); Roldán (1999 y 2003); Castellanos y Serratos (2008). Con relación al departamento del Quindío algunos estudios han sido realizados por Arango y Becerra (1996); Echeverry y Álzate (2003); Giraldo et al. (2008). También se han desarrollado pocos estudios asociados a la composición, estructura numérica, distribución, variaciones temporales y colonización de la comunidad de algas perifíticas, como los reportados por Aragón et al. (2004); Hernández et al. (2005) y Montoya y Ramírez (2007). En el ámbito departamental, Bustamante et al. (2008) estudiaron de manera preliminar la composición y abundancia de la comunidad de microalgas perifíticas del río Quindío.
Teniendo en cuenta que los estudios sobre aspectos ecológicos de comunidades bénticas en las quebradas andinas colombianas son escasos, se pretende ampliar el conocimiento estudiando la composición y estructura numérica de las comunidades bénticas (macroinvertebrados y algas perifíticas) de la quebrada La Florida "Unidad de Manejo de la Cuenca del río Quindío". Lo cual puede ser un aporte significativo para los actores que intervienen en la planificación y gestión integral de los recursos hídricos en el contexto local y regional.

\section{MATERIALES Y MÉTODOS}

\section{Área de estudio}

La quebrada La Florida, se localiza en la zona urbana de la ciudad de Armenia, con coordenadas de ubicación latitud norte 4.55171, longitud oeste -75.66205, coordenadas planas este 1157474.58840 norte 994920.21074 (Figura.1). Esta nace en los límites de Armenia con el municipio de Salento y Circasia vereda La Florida, Punto Colillas, y baja hasta la vereda San Nicolás, donde desemboca al río Quindío. Dentro de la ciudad está delimitada por la avenida Bolívar y la avenida Centenario (Arango y Becerra, 1996).

\section{Estaciones de muestreo}

Basados en el gradiente altitudinal, se ubicaron seis puntos de muestreo distribuidos a lo largo del trayecto longitudinal de la quebrada La Florida, teniendo en cuenta las partes alta, media y baja (Tabla 1 ).

Tabla 1: sitios de muestreo en la quebrada La Florida con su respectiva altitud.

\begin{tabular}{lc}
\hline \multicolumn{1}{c}{ SITIOS } & ALTURA (m.s.n.m) \\
\hline 1- Finca la Irlanda & 1680 \\
2- Puente Chaguala & 1610 \\
3- Sena Agropecuario & 1570 \\
4- La Castellana & 1530 \\
5- Parque de la Vida & 1520 \\
6- Finca Costa Azul & 1290 \\
\hline
\end{tabular}




\section{Toma y procesamiento variables físico-químicas}

Se realizaron cinco jornadas de muestreo, entre los meses de febrero y junio de 2009, teniendo en cuenta los periodos considerados de altas y bajas precipitaciones. En cada uno de los seis sitios se tomaron muestras biológicas y de agua para los análisis físico-químicos, algunas variables se registraron In situ y otras fueron analizadas en el Laboratorio de Aguas y Ambiental de la Universidad del Quindío, siguiendo las recomendaciones de los métodos estandarizados de la "American Public Health Association" (APHA, 1998), (Tabla 2).

Tabla 2: Variables físico-químicas y su respectivo método analítico.

\begin{tabular}{|c|c|}
\hline PARÁMETROS & MÉTODO ANALÍTICO \\
\hline Velocidad de corriente $(\mathrm{m} / \mathrm{s})$ & Cronómetro y pelota de caucho \\
\hline Profundidad (m) & Vara métrica \\
\hline Ancho del cauce (m) & Decámetro \\
\hline Intensidad lumínica (Lux) & Luxómetro (Traceable) Extch instruments foot cande-lux meter \\
\hline Humedad relativa (\%) & Termohigrómetro ficher cientific 11-G51-15 S/N 61602533 \\
\hline Temperatura ambiente (OC) & Termómetro ambiental Silver Brand Germany z-s- 800003 \\
\hline Temperatura del agua (OC) & Termohigrómetro ficher cientific 11-G51-15 S/N 61602533 \\
\hline Conductividad $(\mu \mathrm{S} / \mathrm{cm})$ & Conductímetro HANNA \\
\hline $\mathrm{pH}$ & Potenciómetro HANNA HI99001 \\
\hline Porcentaje de saturación de oxigeno (\%) & Oxímetro handlylab LF1 1 SCHOOT instruments GmbhD-55122 Mai \\
\hline Concentración de oxígeno disuelto $(\mathrm{mg} / \mathrm{L})$ & Método yodométrica (modificación de azida) \\
\hline Demanda bioquímica de oxígeno (mg/L) & Incubación "Estándar" a 5 días \\
\hline Sólidos suspendidos totales (mg/L) & Gavimétrico (Filtración en fibra de vidrio) \\
\hline Turbiedad (FAU) & Turbidímetro (Lovibond Pc Spectron Ahas) \\
\hline Dureza total (mg/L) & Titulométrico con EDTA speedsafe ${ }^{\mathrm{TM}}$ by HANNA \\
\hline Alcalinidad total $(\mathrm{mg} / \mathrm{L})$ & Titulación potenciómetro speedsafe ${ }^{\mathrm{TM}}$ by HANNA \\
\hline
\end{tabular}

\section{Toma y conservación de muestras biológicas}

Las muestras de perifiton se tomaron a partir de raspado y succión, utilizando espátulas y goteros en los diferentes sustratos naturales epifítico (vegetal) y epilítico (roca) elegidos al azar en la orilla y centro de la quebrada. La ubicación de la toma de la muestras fue vertical con relación a la corriente, según lo recomendado por Ramírez (2006). El material encontrado se depositó en recipientes oscuros donde se fijó con lugol al $10 \%$ y posteriormente fueron rotulados para su identificación y recuento microscópico. Las muestras de macroinvertebrados se tomaron utilizando pinzas y una red en forma de $D$, en los diferentes sustratos epifítico (vegetal) y epilítico (roca) elegidos al azar en la orilla y centro de la quebrada. Los especímenes se preservaron en alcohol al $70 \%$ en recipientes plásticos transparentes rotulados para su posterior separación e identificación. Las muestras biológicas se recolectaron en una longitud de $20 \mathrm{~m} 2$ de largo por el ancho de la quebrada con un esfuerzo de muestreo de 1 hora por hombre red en cada sitio.

Rev. Invest. Univ. Quindío (22): 58- 69. Armenia - Colombia

\section{Identificación y conteo de las muestras biológicas}

La identificación de la comunidad de perifiton se efectuó con la ayuda de un microscopio (NIKON E-200) con aumentos 40X y 100X. Para la cuantificación de la densidad de individuos por mililitro (Ind./ml), se aplicó la expresión estandarizada por Ros (1979) citado por Ramírez (2000). Para la determinación taxonómica de la comunidad fitoperifítica se consultaron claves dicotómicas, descripciones e ilustraciones para microalgas de agua dulce de: Sze (1997); Acleto y Zúñiga (1998); APHA, AWWA, WPCF (1998); Ramírez (2000) y Lobo et al. (2004). La identificación de la comunidad de macroinvertebrados se efectuó con la ayuda de un estereoscopio binocular (Leica zoom 2000), y para su identificación taxonómica se emplearon claves dicotómicas, descripciones e ilustraciones de: Pennak (1989); Merritt y cummnis (1996); Roldán (1996); Domínguez y Fernández (2001) y Roldan (2003). 


\section{Análisis ecológico}

Para describir las comunidades bénticas en los diferentes puntos de muestreo, se emplearon los índices de diversidad alfa $(\alpha)$ : Shannon - Wiener $\left(H^{\prime}\right)$, dominancia de Simpson, equidad de Pielou y riqueza ecológica de Margalef; y como índice de diversidad beta ( $\beta)$ : el coeficiente de similaridad de Jaccard.

\section{Análisis de la información y tratamiento estadístico}

Para determinar los índices ecológicos de diversidad alfa $(\alpha)$ se empleó el programa DIVERS y el índice de diversidad beta ( $\beta$ ); con el programa PAST, finalmente se empleó una prueba de Kruskal Wallis para comparar la diversidad ecológica alfa $(\alpha)$, con los sustratos, los sitios y tiempo de muestreo, las diferencias significativas se denotaron con una prueba de medias con el programa STATISTICA versión 7.

\section{RESULTADOS Y DISCUSIÓN}

\section{Composición y estructura comunidad de Macroinvertebrados.}

La comunidad de macroinvertebrados de la quebrada La Florida, estuvo representada por 4 clases, 11 órdenes, 25 familias y 32 géneros, con un total de 12.113 individuos colectados. La clase más representativa fue la Insecta con una abundancia relativa de $90.2 \%$ distribuidos en 8 órdenes, 22 familias y 29 géneros, según lo argumentado por Roldán (1992), esto posiblemente se debe a que estos organismos constituyen una de las clases más complejas, abundantes y mejor distribuidas en el mundo. Las clases menos representativas fueron Nematomorpha, Hirudinea y Oligochaeta cada una representada por 1 orden, 1 familia y 1 género. Estos resultados son similares a los reportados por Arango y Becerra (1996), en esta misma microcuenca.

Tabla 4: Número de taxones, abundancia e índices de diversidad alfa ( $\alpha$ ) de la comunidad de macroinvertebrados asociados a los sustratos epifítico (vegetal) y epilítico (roca).

\begin{tabular}{|c|c|c|c|c|c|c|c|c|c|}
\hline \multirow[b]{2}{*}{ SUSTRATO } & \multirow{2}{*}{$\begin{array}{l}\text { Número } \\
\text { de } \\
\text { ordsenes }\end{array}$} & \multirow{2}{*}{$\begin{array}{c}\text { Número } \\
\text { de } \\
\text { familias }\end{array}$} & \multirow{2}{*}{$\begin{array}{c}\text { Número } \\
\text { de } \\
\text { géneros }\end{array}$} & \multirow{2}{*}{$\begin{array}{l}\text { Número de } \\
\text { individuos }\end{array}$} & \multirow{2}{*}{$\begin{array}{l}\text { Abundancia } \\
\text { relativa (\%) }\end{array}$} & \multirow{2}{*}{$\begin{array}{c}\begin{array}{c}\text { Diversidad } \\
\text { de Shanon } \\
\text { wiener }\end{array} \\
\mathrm{H}^{\prime} \\
\end{array}$} & \multirow{2}{*}{$\begin{array}{c}\begin{array}{c}\text { Equidad } \\
\text { de Pielou }\end{array} \\
E^{\prime}\end{array}$} & \multirow{2}{*}{$\begin{array}{c}\begin{array}{c}\text { Riqueza de } \\
\text { Margalef }\end{array} \\
\text { D Mg } \\
\end{array}$} & \multirow[t]{2}{*}{$\begin{array}{c}\text { Dominancia } \\
\text { de Simpson } \\
\lambda\end{array}$} \\
\hline & & & & & & & & & \\
\hline Epilítico & 10 & 22 & 29 & 5795 & 47.8 & 1.84 & 0.65 & 3.23 & 0.21 \\
\hline Epifítico & 9 & 14 & 16 & 6318 & 52.1 & 1.82 & 0.54 & 1.71 & 0.26 \\
\hline \multicolumn{4}{|c|}{ TOTAL } & 12113 & 100 & & & & \\
\hline
\end{tabular}

El sustrato epilítico registró los máximos valores de diversidad con 1.84 , de equidad con 0.65 y de riqueza con 3.23; y presentó los valores mínimos de abundancia relativa con $47.8 \%$ y de dominancia con 0.21 (Tabla 4). No obstante la prueba estadística de Kruskal Wallis solo registró diferencias significativas entre los sustratos con respecto a la equidad ( $\mathrm{H}: 3,938182 ; \mathrm{g}=10 ; \mathrm{p}=0.0472)$.

Con base a Roldán (1992), se puede afirmar que el sustrato epilítico muestra tendencia a ser el refugio preferido por la comunidad de macroinvertebrados, ya que este alberga gran variedad de organismos y puede ofrecer más estabilidad y seguridad, mientras el sustrato epifítico (material vegetal) es menos estable, puesto que pueda ser arrastrado por las corrientes más rápidas.

Los sustratos presentaron una similitud del $40 \%$ (Figura 2), compartiendo 13 de las 32 morfoespecies de macroinvertebrados, como Placobdella sp., Physa sp., Simulium sp., Protóptila sp., Smicridea sp.,Stridulivelia sp., Neochordodes sp., Tendipes sp., Típula sp., Ferrisia sp., Baetodes sp., Leptonema sp. y Psephenops sp.

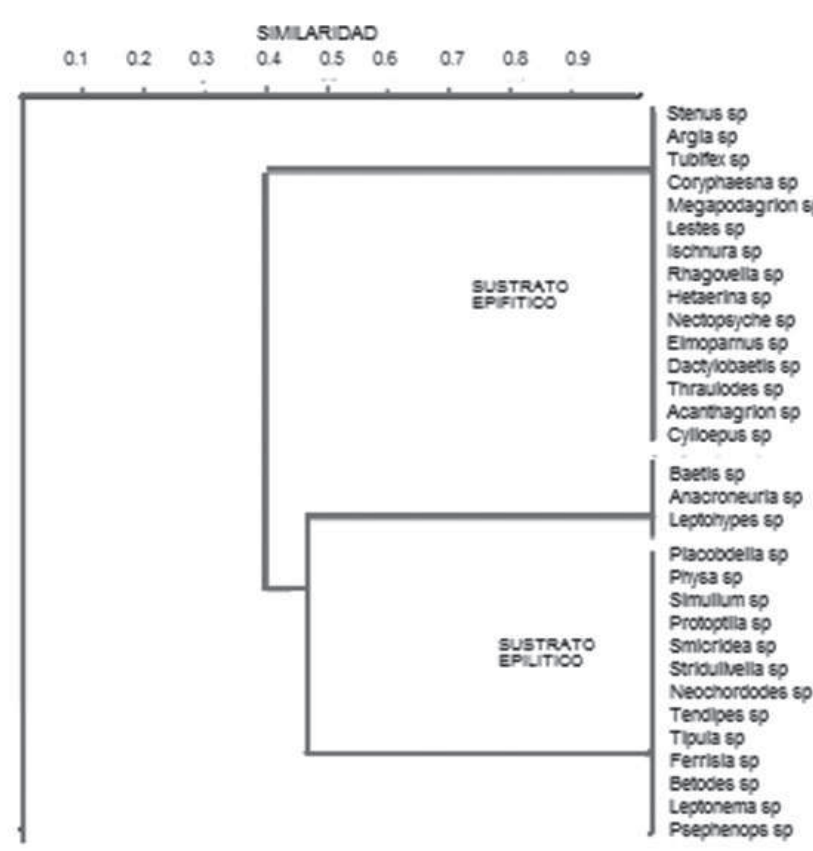

Figura 1: Dendograma de similitud entre los sustratos epifítico (vegetal) y epilítico(roca) para la comunidad de macroinvertebrados. 
Según Domínguez y Fernández (2001), estos macroinvertebrados se pueden encontrar principalmente adheridos a la vegetación y debajo de las piedras.

Tabla 5: Número de taxones, abundancia y diversidad alfa $(\alpha)$ de la comunidad de macroinvertebrados en los diferentes meses de muestreo.

\begin{tabular}{|c|c|c|c|c|c|c|c|c|c|}
\hline MESES & $\begin{array}{c}\text { Número } \\
\text { de } \\
\text { ordenes }\end{array}$ & $\begin{array}{c}\text { Número } \\
\text { de } \\
\text { familias }\end{array}$ & $\begin{array}{c}\text { Número } \\
\text { de } \\
\text { géneros }\end{array}$ & $\begin{array}{c}\text { Número } \\
\text { de } \\
\text { individuos }\end{array}$ & $\begin{array}{l}\text { Abundancia } \\
\text { relativa (\%) }\end{array}$ & $\begin{array}{c}\text { Diversidad } \\
\text { de Shanon } \\
\text { wiener H' }\end{array}$ & $\begin{array}{c}\text { Equidad } \\
\text { de } \\
\text { Pielou } \\
\text { E' }^{\prime} \\
\end{array}$ & $\begin{array}{c}\text { Riqueza } \\
\text { de } \\
\text { Margalef } \\
\text { DMg } \\
\end{array}$ & $\begin{array}{c}\text { Dominancia } \\
\text { de Simpson } \\
\lambda\end{array}$ \\
\hline FEBRERO & 11 & 17 & 21 & 2701 & 22.3 & 1.86 & 0.63 & 2.53 & 0.22 \\
\hline MARZO & 10 & 17 & 17 & 2482 & 20.49 & 1.85 & 0.61 & 2.04 & 0.20 \\
\hline ABRIL & 7 & 9 & 9 & 2218 & 18.28 & 1.76 & 0.60 & 1.93 & 0.22 \\
\hline MAYO & 8 & 11 & 11 & 2321 & 19.16 & 1.77 & 0.60 & 1.92 & 0.23 \\
\hline JUNIO & 7 & 12 & 13 & 2391 & 19.73 & 1.74 & 0.58 & 1.92 & 0.23 \\
\hline \multicolumn{4}{|c|}{ TOTAL } & 12113 & 100 & & & & \\
\hline
\end{tabular}

El mes de febrero registró los máximos valores de abundancia relativa con $22.2 \%$, de diversidad con 1.86 , de equidad con 0.63 y de riqueza con 2.53 (Tabla 5). Dicho mes es considerado de bajas Iluvias para la zona cafetera colombiana, por lo tanto, en este periodo hidroclimático no se evidencian cambios desfavorables en la dinámica y estructura ecológica de los macroinvertebrados bentónicos, por el contrario, este periodo seco puede favorecer a que los hábitats y refugios de estos organismos sean más estables y no estén expuestos a ser arrastrados aguas abajo del cauce (deriva natural) como consecuencia de las lluvias.

Entre tanto, los meses de abril y mayo que son considerados de lluvia, y junio considerado de transición hidroclimático, presentaron los valores mínimos de abundancia relativa con $18.28 \%, 19.16 \%$ y $19.73 \%$, de diversidad con $1.76,1.77$ y 1.74 , de equidad con $0.60,0.60$ y 0.58 y de riqueza con $1.93,1.92$ y 192 , respectivamente. Aunque los resultados estadísticos de la prueba Kruskal Wallis, no presentaron diferencias significativas ( $P>0.05$ ) para la diversidad alfa de los macroinvertebrados entre los meses de muestreo.

Los meses de muestreo de la comunidad de macroinvertebrados en la quebrada La Florida, presentaron una similitud de $28 \%$ (Anexo 1), donde agruparon 9 de las 32 morfoespecies registrados como: Leptonema sp., Lestes sp., Anacroneuria sp., Limonia sp., cylloepus sp., Argia sp., Thraulodes sp., Stenus sp. y Psephenops sp., La presencia de estos organismos fue más significativa en los periodos considerados húmedos (marzo y abril), según lo dicho por Roldán (1992) esto se debe a que la mayoría de estos organismos son considerados buenos nadadores, por ende su supervivencia no se ve afectada por los procesos de deriva natural, generado principalmente por las precipitaciones.

Con relación a la distribución de los macroinvertebrados en la quebrada La Florida (Tabla 6), En el sitio 1 se registraron los mayores valores de diversidad con 2.32, de equidad con 1.79, y de riqueza con 3.02; también se registraron los valores mínimos de dominancia con 0.14 y de abundancia relativa con $3.19 \%$. Lo cual indican que en este lugar, las condiciones físico-químicas son favorables para albergar variedad de taxas, por tanto este sitio denota buenas condiciones ecológicas; según Roldán (2003), una comunidad cuando no está bajo la presión de la polución se caracteriza por tener un alto número de especie y un bajo número de individuos por especie.

Situación contraria ocurre con los sitios 4, 5 y 6, parte media y baja de la quebrada La Florida, que registraron los valores mínimos de diversidad con $1.61,1.53$ y 1.57 , de equidad con $0.62,0.66$ y 0.67 , y de riqueza con 1.11 , 1.00 y 0.98 respectivamente, pero registraron los máximos valores de abundancia relativa con $23,46 \%$, $25.88 \%$ y $27.46 \%$, y de dominancia con $0.23,0.24$ y 0.25 respectivamente. Esto coincide en parte con los resultados estadísticos de la prueba Kruskal Wallis, donde solo se registraron diferencias significativas para las abundancias entre dichos sitios $(\mathrm{H}: 25,20129, \mathrm{gL}=30$; $p=0.0001$ ). Dicho aspecto puede obedecer a que la mayoría de los sitios presentan condiciones diferentes en cuanto a caudal, calidad de agua, disponibilidad de sustratos y cobertura ribereña. 
Tabla 6: Número de taxones, abundancia y diversidad alfa $(\alpha)$ de la comunidad de macroinvertebrados en los sitios de muestreo.

\begin{tabular}{|c|c|c|c|c|c|c|c|c|c|}
\hline SITIOS & ORDEN & FAMILIA & GENERO & $\begin{array}{c}\text { \#DE } \\
\text { INDIVIDUOS }\end{array}$ & $\begin{array}{l}\text { ABUNDANCIA } \\
\text { RELATIVA } \%\end{array}$ & $\begin{array}{c}\text { Diversidad } \\
\text { de Shanon } \\
\text { Wiener }\end{array}$ & $\begin{array}{c}\begin{array}{c}\text { Equidad } \\
\text { de Pielou }\end{array} \\
E^{\prime}\end{array}$ & $\begin{array}{c}\text { Riqueza } \\
\text { de } \\
\text { Margalef }\end{array}$ & $\begin{array}{l}\text { Dominancia } \\
\text { de Simpson }\end{array}$ \\
\hline 1.Finca La Irlanda & 7 & 17 & 19 & 387 & 3.19 & 2.32 & 1.79 & 3.02 & 0.14 \\
\hline 2.Puente Chagualá & 7 & 11 & 12 & 507 & 4.18 & 2.01 & 0.80 & 1.76 & 0.17 \\
\hline 3.Sena Agropecuario & 8 & 14 & 14 & 1915 & 15.8 & 1.65 & 0.73 & 1.72 & 0.23 \\
\hline 4.La Castellana & 6 & 9 & 9 & 2842 & 23.46 & 1.61 & 0.62 & 1.11 & 0.23 \\
\hline 5.Parque de la Vida & 5 & 9 & 9 & 3135 & 25.88 & 1.53 & 0.66 & 1.00 & 0.24 \\
\hline 6. Finca Costa Azul & 7 & 9 & 9 & 3327 & 27.46 & 1.57 & 0.67 & 0.98 & 0.25 \\
\hline
\end{tabular}

El porcentaje de similitud de los macroinvertebrados entre los sitios de muestreo fue muy bajo (Anexo 2), de solo $3 \%$; pues de las 32 morfoespecies registradas solo agruparon una que fue Simulium sp. Los 2 primeros sitios tuvieron una similitud de $13 \%$, compartiendo estas morfoespecies: Lestes sp., Protóptila sp., Smicridae sp., Megapodagrion sp. y Leptohypes sp. Mientras los sitios 3, 4,5 y 6 tuvieron una semejanza del $10 \%$, pues solo agruparon 3 de las 32 morfoespecies registradas como: Tendipes sp., Placobdella sp., y Típula sp. Estos resultados son algo similares a los reportados por Downes et al. (2000) quienes afirman que los sitios en un mismo río o quebrada pueden presentar una composición taxonómica de macroinvertebrados muy diferente. Asimismo Heino et al. (2004) dicen que la composición de macroinvertebrados en diferentes locaciones son más variables en las pequeñas cabeceras de ríos que en los río de mayor orden, como sucede en este caso.

\section{Composición y estructura comunidad fitoperifítica}

La comunidad fitoperifítica de la quebrada La Florida, estuvo representada por 4 divisiones, 26 familias y 42 géneros con un total de 23.626 (Ind/ml). La división más representativa fue Bacillariophyta con una abundancia relativa de $58.70 \%$, estos resultados son similares a los reportados por Bustamante et al. (2008). Las Bacillariophyta presentan características morfo-fisiológicas diferentes a las demás, como es la presencia de pared celular silicada, tienen finas gotas de aceite las cuales son reservas nutritivas, y una alta perdurabilidad de la membrana; todas estas características brindan protección y un amplio rango de tolerancia a muchos factores de vulnerabilidad, lo que hace que la mayoría de sus representantes puedan sobrevivir en diferentes ambientes acuáticos (Lewin et al., 1977 citado de Hernández et al., 2005).

La segunda división más representativa fue Chlorophyta con una abundancia relativa de $25.28 \%$, distribuidas en 10 familias y 19 géneros; seguida de la división Cyanophyta con 6 familias, 9 géneros y una abundancia relativa de $15.13 \%$; finalmente la división Euglenophyta fue la menos representativa con 2 familias, 2 géneros y 205 Ind./ml y una abundancia relativa de $0.86 \%$.

Tabla 7: Número de taxones, abundancia y diversidad alfa $(\alpha)$ de la comunidad de fitoperifítica asociada los sustratos epifítico (vegetal) y epilítico (roca).

\begin{tabular}{|c|c|c|c|c|c|c|c|c|c|}
\hline \multirow[b]{2}{*}{ SUSTRATO } & \multirow[b]{2}{*}{ DIVISION } & \multirow[b]{2}{*}{ FAMILIA } & \multirow[b]{2}{*}{ GENERO } & \multirow{2}{*}{$\begin{array}{c}\text { \# DE } \\
\text { INDIVIDUOS }\end{array}$} & \multirow{2}{*}{$\begin{array}{l}\text { ABUNDANCIA } \\
\text { RELATIV A (\%) }\end{array}$} & \multirow{2}{*}{$\begin{array}{c}\text { Diversidad } \\
\text { de Shanon } \\
\text { Wiener }\end{array}$} & \multirow{2}{*}{$\begin{array}{c}\begin{array}{c}\text { Equidad } \\
\text { de } \\
\text { Pieluo }\end{array} \\
\text { E' }^{\prime}\end{array}$} & \multirow{2}{*}{$\begin{array}{c}\begin{array}{c}\text { Riqueza } \\
\text { de } \\
\text { Margalef }\end{array} \\
D_{\mathrm{Mg}}\end{array}$} & \multirow{2}{*}{$\begin{array}{c}\text { Dominancia } \\
\text { de Simpson } \\
\lambda\end{array}$} \\
\hline & & & & & & & & & \\
\hline Epifítico & 4 & 23 & 34 & 9786 & 41.42 & 1.70 & 0.48 & 3.59 & 0.26 \\
\hline \multirow[t]{2}{*}{ Epilítico } & 4 & 21 & 29 & 13840 & 58.57 & 1.62 & 0.39 & 2.93 & 0.28 \\
\hline & \multicolumn{2}{|c|}{ TOTAL } & & 23626 & 100 & & & & \\
\hline
\end{tabular}


Respecto a la estructura numérica del fitoperifiton asociada a los sustratos (Tabla 7), el sustrato epifítico registró los valores máximos de diversidad con 1.70 , de equidad con 0.48 , y de riqueza con 3.59 , y registró los valores mínimos de abundancia relativa con $41.42 \%$, y de dominancia con 0.26 . Por su parte, el sustrato epilítico registró los valores máximos de abundancia relativa con $58.57 \%$ y de dominancia con 0.28 ; y presentó los valores mínimos de diversidad con 1.62, de equidad con 0.39 , y de riqueza con 2.93. Pero de acuerdo a los resultados estadísticos de la prueba de Kruskall Wallis no se presentaron diferencias significativas para la estructura numérica del fitoperifiton entre los sustratos ( $p>0.05$ ).

En términos generales, la literatura reporta que el hábitat más apropiado para albergar a la comunidad de perifiton es el material vegetal, ya que puede retener mayor cantidad de nutrientes necesarios para la supervivencia de estos individuos otorgando con más facilidad refugio y alimento (Sze, 1997 y Ramírez, 2000).

Los sustratos presentaron una similitud del $50 \%$ (Figura 2), pues solo comparten 21 de las 42 morfoespecies registradas de fitoperifiton, esto es debido principalmente a su $\mathrm{A}$ a que estas especies tienen una alta adaptabilidad, gracias a la presencia de tricomas móviles que facilitan el desplazamiento entre los sustratos, permitiendo que este sea muy variado considerándose cosmopolitas (Acleto y Zúñiga, 1998).

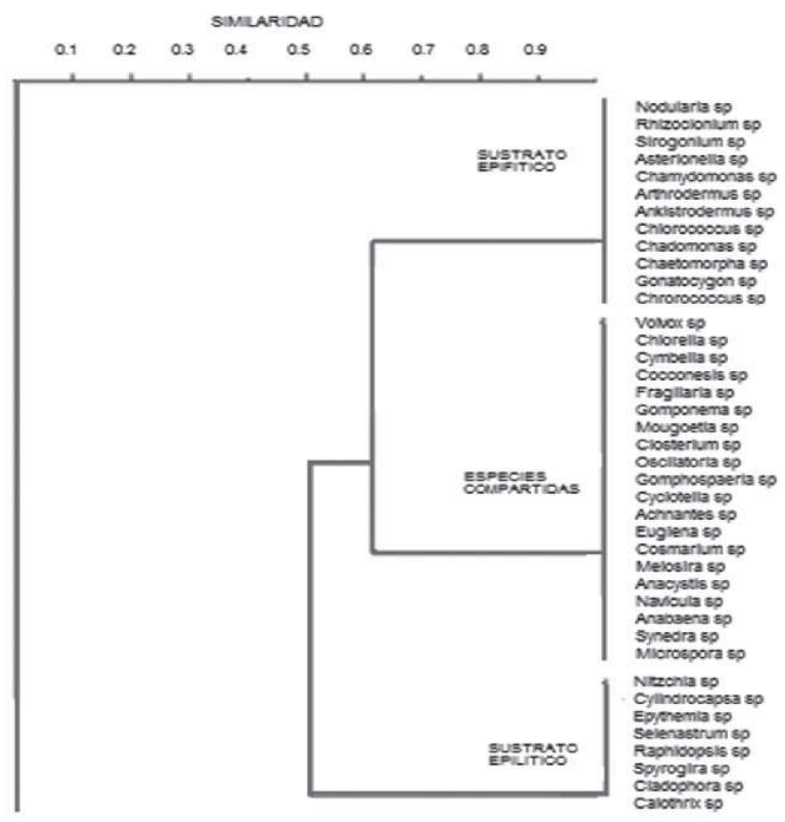

Figura 2: Dendograma de similitud entre los sustratos epifítico (material vegetal) y epilíctico (roca) para la comunidad fitoperifítica.

Los valores sobre composición taxonómica y diversidad alfa para la comunidad fitoperifítica entre los meses muestreados fueron muy homogéneos (Tabla 8), lo cual se corrobora con los resultados estadísticos de la prueba de Kurskall Wallis que no registró diferencias significativas ( $P>0.05)$.

Tabla 8: Número de taxones, abundancia y diversidad alfa $(\alpha)$ de la comunidad de fitoperifiton en los diferentes meses de muestreo.

\begin{tabular}{lccccccccc}
\hline MESES & DIVISION & FAMILIA & GENERO & $\begin{array}{c}\text { \# DE } \\
\text { INDIVIDUOS }\end{array}$ & $\begin{array}{c}\text { ABUNDANCIA } \\
\text { RELATIVA (\%) }\end{array}$ & $\begin{array}{c}\text { Diversidad } \\
\text { de Shanon } \\
\text { Wiener H' }\end{array}$ & $\begin{array}{c}\text { Equidad } \\
\text { de } \\
\text { Pielou E' }\end{array}$ & $\begin{array}{c}\text { Riqueza } \\
\text { de } \\
\text { Margalef } \\
\mathbf{D}_{\text {Mg }}\end{array}$ & $\begin{array}{c}\text { Dominancia } \\
\text { de Simpson } \\
\boldsymbol{\lambda}\end{array}$ \\
\hline FEBRERO & 4 & 17 & 19 & 4161 & 17.61 & 1.76 & 0.54 & 2.87 & 0.24 \\
MARZO & 4 & 17 & 18 & 3625 & 15.34 & 1.75 & 0.53 & 2.86 & 0.25 \\
ABRIL & 4 & 16 & 19 & 3730 & 16.71 & 1.76 & 0.54 & 2.87 & 0.24 \\
MAYO & 4 & 15 & 16 & 3545 & 15.22 & 1.75 & 0.56 & 2.79 & 0.24 \\
JUNIO & 4 & 16 & 17 & 4565 & 19.32 & 1.75 & 0.54 & 2.86 & 0.25 \\
& \multicolumn{1}{l}{ TOTAL } & & & 23626 & 100 & & & & \\
\hline
\end{tabular}


Según la distribución de las algas perifíticas en la quebrada La Florida (Tabla 9), los sitios 1 y 2 que son parte alta de la microcuenca, registraron los valores máximos de diversidad con 1.47 , de equidad con 0.53 y 0.49 , y de riqueza con 2.67 y 2.34 respectivamente; $y$ presentaron los valores más bajos de dominancia con 0.20 y 0.22 respectivamente. Dichos sitios ofrecen una mayor cantidad y variedad de microhábitats para el desarrollo del fitoperifiton, lo cual probablemente haya favorecido su riqueza y diversidad en comparación con las demás localidades evaluadas.

Los sitios 4,5 y 6 presentaron los valores mínimos de diversidad con $1.30,1.03$ y 1.24 , de equidad $0.35,0.43$ y 0.39 , y de riqueza con 1.06, 1.14 y 1,12 respectivamente; y presentando los valores máximos de dominancia con $0.26,0.40$ y 0,46 respectivamente. Estos resultados coinciden en parte con los estadísticos (Kruskal Wallis), donde solo se presentaron diferencias significativas con respecto a las abundancias entre los sitios 1,5 y $6(\mathrm{H}: 12,27529$; $g L=30 ; p=0.0312$ ).

Los resultados de similitud entre los meses de muestreo para la comunidad fitoperifítica fue de $21 \%$ (Anexo 3), los meses muestreados solo agruparon 9 de las 42 morfoespecies identificadas, entre estas: Navícula sp., Fragilaria sp, Melosira sp., Chlorella sp., Anabaena sp., Euglena sp., Synedra sp., Closterium sp. y Oscillatoria sp. Estas algas perifíticas tienen una alta tasa reproductiva, compensando así la perdidas de arrastre generadas posiblemente por las corrientes causadas por los periodos de altas lluvias (Sze, 1997).

Tabla 9: Número de taxones, abundancia y diversidad alfa $(\alpha)$ de la comunidad de fitoperifiton en los sitios de muestreo.

\begin{tabular}{|c|c|c|c|c|c|c|c|c|c|c|}
\hline SITIOS & DIVISION & FAMILIA & A GENERO & $\begin{array}{c}\text { \# DE } \\
\text { INDIVIDUO }\end{array}$ & & $\begin{array}{l}\text { BUNDANCIA } \\
\text { BELATIVA (\%) }\end{array}$ & $\begin{array}{c}\text { Diversidad } \\
\text { de Shannon } \\
\text { Wiener H' }\end{array}$ & $\begin{array}{l}\text { Equidad de } \\
\text { Pielou E' }\end{array}$ & $\begin{array}{c}\text { Riqueza de } \\
\text { Margalef } \\
D_{\mathrm{Mg}} \\
\end{array}$ & $\begin{array}{c}\text { Dominancia } \\
\text { de Simpson } \\
\lambda\end{array}$ \\
\hline \multicolumn{2}{|c|}{ 1.Finca La Irlanda } & 3 & 14 & 16 & 2465 & 10.4 & $\begin{array}{ll}43 & 1.47\end{array}$ & 0.53 & 2.67 & $\begin{array}{ll}7 & 0.20\end{array}$ \\
\hline \multicolumn{2}{|c|}{ 2.Puente Chagualá } & 3 & 13 & 15 & 3335 & 14.1 & 1.47 & 0.49 & 2.34 & 0.22 \\
\hline \multicolumn{2}{|c|}{ 3.Sena Agropecuario } & 3 & 10 & 13 & 3535 & 14.9 & 1.34 & 0.45 & 1.83 & 0.24 \\
\hline \multicolumn{2}{|c|}{ 4.La Castellana } & 4 & 10 & 13 & 3795 & 16.0 & 1.30 & 0.35 & 1.06 & 0.26 \\
\hline \multicolumn{2}{|c|}{ 5.Parque de la Vida } & 4 & 10 & 11 & 5079 & 21.4 & 1.03 & 0.43 & 1.14 & 0.40 \\
\hline \multirow{2}{*}{\multicolumn{2}{|c|}{ 6.Finca Costa Azul }} & 4 & 9 & 11 & 5417 & 22.9 & 1.24 & 0.39 & 1.12 & 0.46 \\
\hline & & TOTAL & & & 23626 & 10 & & & & \\
\hline
\end{tabular}

El porcentaje de similitud para la comunidad fitoperifítica entre los sitios de muestreo (Anexo 4) fue apenas del 7\%, dichos sitios solo compartieron 3 de las 42 morfoespecies como: Chlorella sp., Navícula sp. y Sinedra sp. Estos valores bajos del porcentaje de similitud indican una variabilidad significativa en la composición de morfoespecies fitoperifíticas en los sitios de la quebrada La Florida.

\section{Variables físico-químicas}

Los análisis estadísticos descriptivos de las variables físico-químicas, muestra que un grupo de estas, registraron valores promedios que se encuentran dentro de los rangos establecidos de buena calidad para este tipo de sistemas lóticos de la ecorregión andina como son la conductividad, dureza, $\mathrm{pH}$, alcalinidad, sólidos suspendidos y temperatura (Tabla 10), que corresponden a aguas oligotróficas, poco duras y casi neutras, lo cual favorecen el crecimiento y desarrollo de comunidades bénticas como el perifiton y los macroinvertebrados (Arango y Becerra, 1996; Giraldo et al. 2008; Bustamante et al. 2008). Sin embargo, las variables que presentaron grandes fluctuaciones durante el periodo de estudio fueron la concentración y saturación de oxígeno, turbiedad, sólidos suspendidos, dureza, conductividad y demanda bioquímica de oxígeno, lo que pudo darse como consecuencia de las precipitaciones que causa cambios en el caudal y por el vertimiento de aguas residuales domésticas que alteran las concentraciones de oxígeno, sólidos y materia orgánica, que son patrones condicionantes de la vida acuática (Ramírez, 2006).

Las variables temperatura del agua y ambiente, humedad relativa e intensidad lumínica, fluctuaron poco durante el periodo de estudio, lo que evidencia condiciones microclimáticas estables de la 
Tabla 10: Valores promedios $\overline{(X)}$, coeficiente de variación (CV), mínimas (Min) y máximas (Max) de las variables físico-químicas.

\begin{tabular}{lcccc}
\hline \multicolumn{1}{c}{ VARIABLES } & MIN & MAX & X & CV \\
\hline Alcalinidad $\left(\mathrm{mg} / \mathrm{L} \mathrm{CaCO}_{3}\right)$ & 0 & 54 & 15 & 74 \\
Dureza $\left(\mathrm{mg} / \mathrm{L} \mathrm{CaCO}_{3}\right)$ & 0 & 62 & 23 & 82 \\
Conductividad $(\mu \mathrm{S} / \mathrm{cm})$ & 8,2 & 208 & 48 & 78 \\
$\mathrm{pH}$ & 5,9 & 7,7 & 7,6 & 49 \\
$\mathrm{DBO}_{5}\left(\mathrm{mgO}_{2} / \mathrm{L}\right)$ & 0,43 & 6,7 & 4.3 & 75 \\
Oxígeno disuelto $(\mathrm{mg} / \mathrm{L})$ & 2,1 & 9,9 & 4.4 & 98 \\
Saturación de oxígeno (\%) & 42 & 87 & 70 & 91 \\
Sólidos suspendidos $(\mathrm{mg} / \mathrm{L})$ & 0 & 436 & 65 & 90 \\
Turbiedad (FAU) & 5 & 392 & 73 & 94 \\
Velocidad de corriente $(\mathrm{m} / \mathrm{s})$ & 2,4 & 22 & 6,9 & 66 \\
Profundidad (m) & 9,3 & 56 & 26 & 58 \\
Ancho (m) & 1,3 & 12 & 6,6 & 55 \\
Intensidad lumínica $(\mathrm{Lux})$ & 224 & 735 & 453 & 13 \\
Humedad relativa $(\%)$ & 58 & 96 & 86 & 11 \\
Temperatura del agua $\left({ }^{\circ} \mathrm{C}\right)$ & 17 & 22 & 19 & 7,5 \\
Temperatura ambiente $\left({ }^{\circ} \mathrm{C}\right)$ & 19 & 28 & 22 & 9,6 \\
\hline
\end{tabular}

microcuenca La Florida; en cambio las variables como ancho del cauce, velocidad de corriente y profundidad aumentaron sus valores en función al comportamiento hidroclimático y descenso del gradiente altitudinal de la microcuenca, por ende los sitios 1,2 y 3 se caracterizaron por ser poco profundos y angostos mientras que los sitios 4,5 y 6 fueron más anchos, profundos y corrientosos.

\section{BIBLIOGRAFÍA}

Acleto, O.y Zúñiga, A. (1998). Introducción a las algas. (1ra edición). Lima, Perú. 500pp.

APHA, AWWA, WPCF. (1998). Standard methods, for the examination of water and wastewater. American public health association, Inc. (20th edición). New York.

Arango, A. y Becerra, F. (1996). Evaluación del impacto ambiental y ecológico en la quebrada La Florida afluente del Rio Quindío en el municipio de Armenia (Tesis de grado). Armenia: Facultad de Educación. Universidad del Quindío.

Aragón A., Geova, S. y Murillo, S. (2004). Estudio comparativo sobre la colonización de fitoperifiton en dos sustratos quebrada la Francisca, municipio de Quibdo. institucional. 20: 40-45.

Bustamante, C., Monsalve, E. y García, P. (2008). Análisis de la calidad de agua en la cuenca media del río Quindío con base en índices físicos, químicos y biológicos. Revista de Investigaciones Universidad del Quindio.18:22-31.

Bustamante, C., Dávila, C., Torres, S. y Ortiz, J. (2008). Composición y abundancia de fitoperifiton en el río Quindío. Revista de investigaciones Universidad del Quindío. 18:15-21.

Castellanos, P. y Serrato, C. (2008). Diversidad de macroinvertebrados acuáticos en un nacimiento de río en el Páramo de Santurbán, Norte de Santander. Academia colombiana de ciencias biológicas. 32: 79-86.

Domínguez, E. y Fernández, H. (2001). Guía para la determinación de los artrópodos bentónicos Sudamericanos. (1ra edición).Tucumán, Argentina. 282 pp.

Downes, B., Hindell, J. y Bond, N. (2000). What's in a site variation in lotic macroinvertebrate density and diversity in a spatially replicate experiment. Austral Ecology. 25: 128-139 
Echeverry, A. y Alzate, J. (2003). Caracterización de la comunidad de macroinvertebrados acuáticos del Rio Roble, Quindío (Tesis de grado). Armenia: Facultad de Educación. Universidad del Quindío.

Giraldo, V., Bulla, C. y Bustamante, C. (2008). Diagnóstico de la calidad ambiental del agua para uso agrícola de la quebrada Los Micos afluente microcuenca El Pencil. Revista Asociación Colombiana de Ciencias Biológicas. 20:144-163.

Hernández, E., Aguirre, N. y Palacio, J. (2005). Variación Espacio-temporal de las estructura de la comunidad perifítica en la microcuenca de la quebrada la vega, municipio de San Roque (Antioquia), Colombia. Acta biológica. 27:67-77.

Heino, J., Louhi, P. y Muotka, T. (2004). Identifing the scales of variability in stream macroinvertebrate abundance, functional composition and assemblage structure. Fresh Biology. 49: 1230-1239.

Lobo, E., Callegero, V., Hermany Gomez, N., Ector, L. (2004). Review of the use of microalgae in south America for monitoring rivers, with special reference to diatoms. Vie et Milieu, France. V.53 N.2/3: 35-45.

Machado, T. y Roldán, G. (1981). Estudio de las características físico-químicas y biológicas del río Anori y sus principales afluentes. Acta biológica. 10:3-19.

Merritt, R. y Cummins, Z. (1996). An Introduction to the Aquatic Insects of North America. (3ra edicion), New York. $862 \mathrm{pp}$.

Montoya, Y. y Ramírez, J. (2007). Variación en la estructura de la comunidad perifítica colonizadora de sustratos artificiales en dos estaciones de muestreo en la zona de litoral del río Medellín. Biología tropical. 55: 585- 593.

Pennak, R. (1989) Freshwater invertebrates of United States. Third edition. Wiley-Interscience, New York. 628 pp.

Ramírez, J. (2000). Fitoplancton de agua dulce: aspectos ecológicos, taxonómicos y sanitarios. (1ra edición). Universidad del Antioquia. Medellín Colombia. 226pp.

Ramírez, A. (2006). Ecología Métodos de muestreo y análisis de poblaciones y comunidades. (1ra edición). Bogotá Colombia. 302pp.

Roldán, G., Builes, J., Trujillo, C. y Suarez, A. (1973). Efectos de la contaminación industrial y doméstica sobre la fauna béntica del río Medellín. Acta biológica. 2:54-64.

Roldán, G. (1992). Fundamentos de Limnología Neotropical. (1ra edición). Universidad de Antioquia. Medellín Colombia. 529pp.

Roldán, G. (1996). Guía para el estudio de los macroinvertebrados acuáticos del departamento de Antioquia. (1ra edición). Universidad de Antioquia. Medellín Colombia. 217pp.

Roldán, G. (2003). Bioindicación de la calidad del agua en Colombia. Propuesta para el uso del método BMWP/Col. Ciencia y Tecnología. (1ra edición) Medellín Colombia. 170 pp.

Sze, P. (1997). A Biology of the Algae. (3ra edición). New York. 500pp. 


\section{ANEXOS}

Diversidad beta comunidad de macroinvertebrados acuáticos

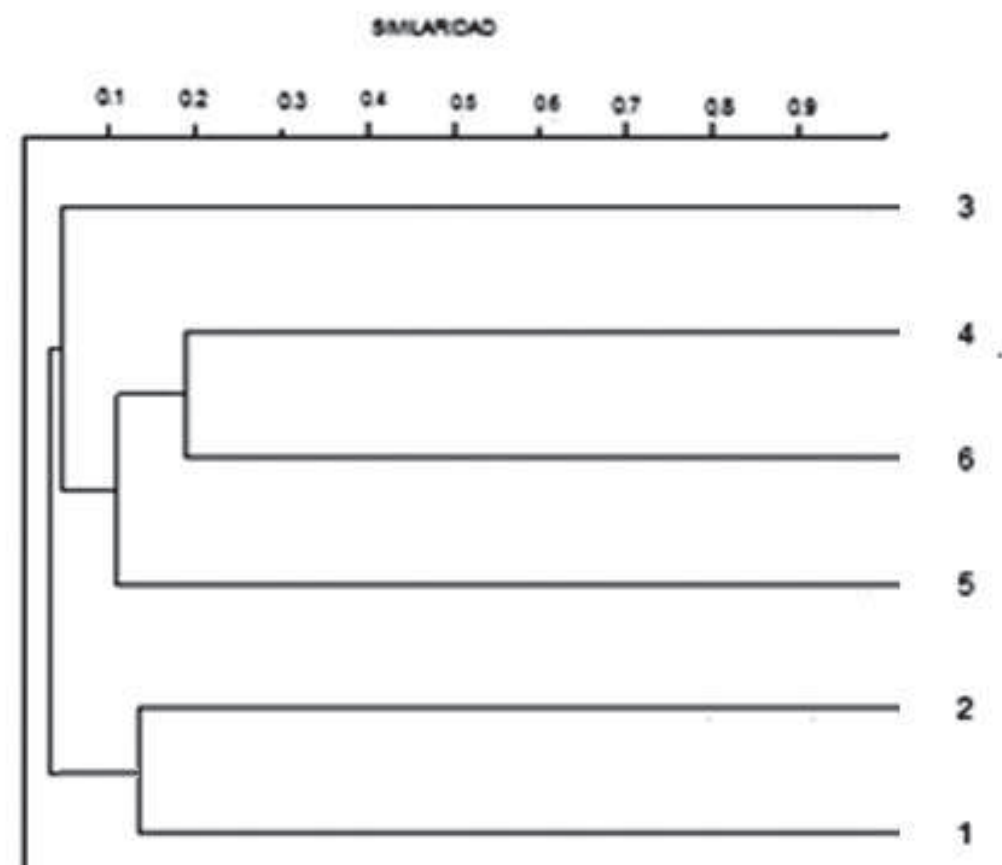

1. Dendograma de similitud entre los meses de muestreo comunidad de macroinvertebrados.

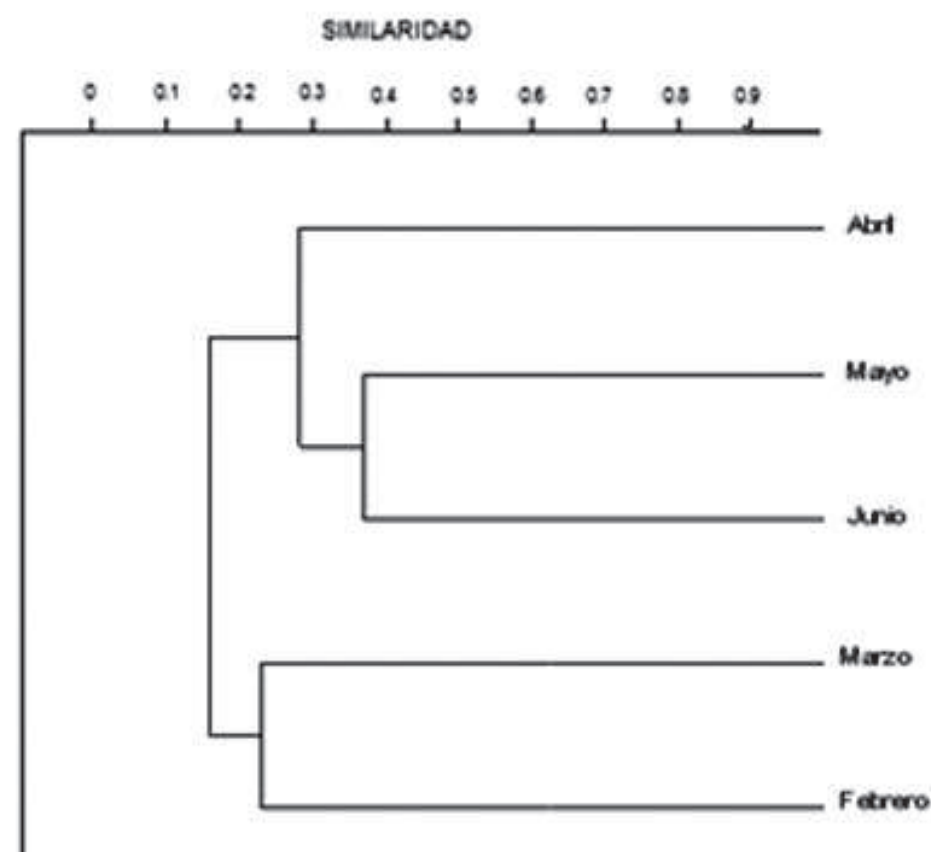

2. Dendograma de similitud entre los sitios de muestreo comunidad de macroinvertebrados. 


\section{ANEXOS}

Diversidad beta comunidad de macroinvertebrados acuáticos

sarunce:

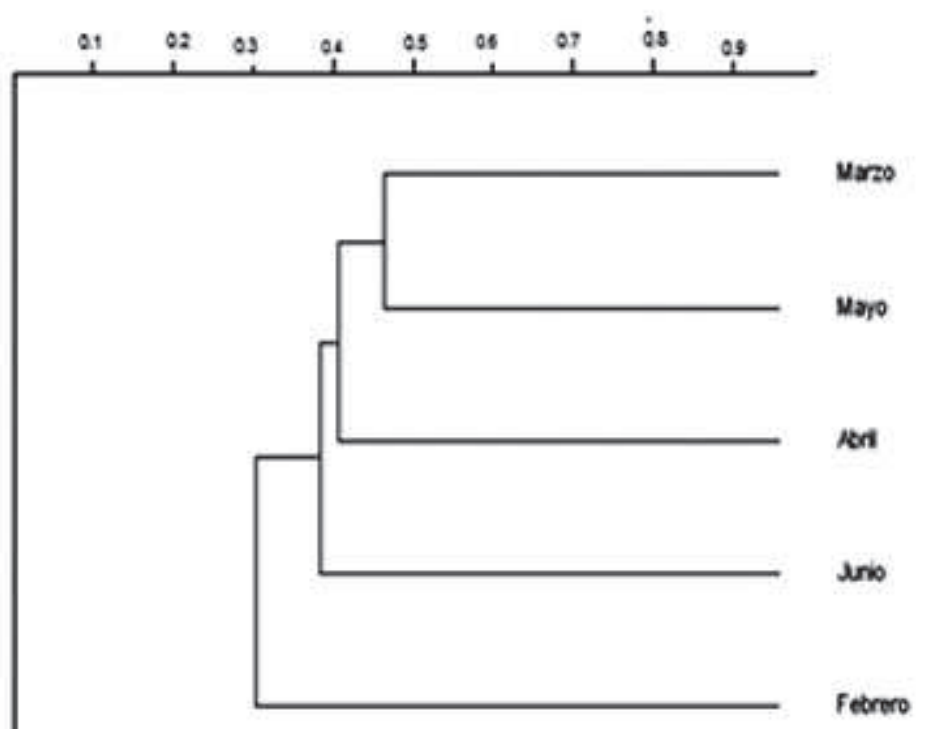

2. Dendograma de similitud entre los meses de muestreo fitoperifiton. suruncos

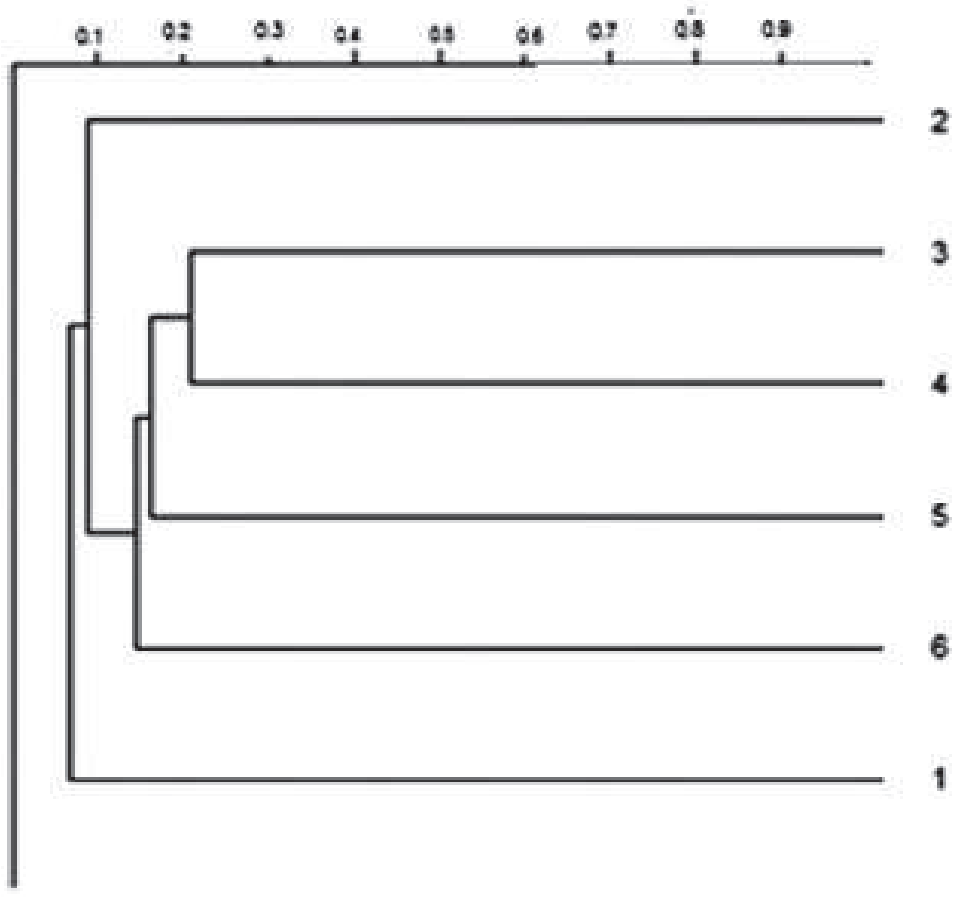

1. Dendograma de similitud entre los meses de muestreo fitoperifiton. 